\title{
SUPER SIZE ME
}

\section{Erhard Taverna}

Sich im Kino zu Tode zu fressen war schon einmal ein Thema. Als schwarze Komödie 1973 mit Mastroianni, Piccoli, Noiret und Tognazzi in «La grande bouffe». Schamlos, vulgär und lustvoll bringen es vier Männer im besten Alter hinter sich. Obwohl unerbittlich derb und direkt erzählt, hatte die Geschichte tragisch-melancholische Züge und einzelne Szenen waren von kaum zu überbietender Komik.

\section{Der Film}

Der New Yorker Filmemacher Morgan Spurlock dokumentiert 2004 einen heroischen Selbstversuch, der an die Tradition jener Mikrobiologen erinnert, die vor Publikum ihre Reagenzröhren austranken. Dreissig Tage hat er sich ausschliesslich McDonald's-Produkte einverleibt. Mindestens dreimal täglich, wenn möglich die grössten Portionen, ein Jäger im Techno-Junk-Food-Land. Drei Mediziner, eine Ernährungsberaterin und ein Sportlehrer attestierten ihm beste Gesundheit und Kondition, bevor er sich mit Schrittzähler und Kamera daranmachte, das Drehbuch zur hyperkalorischen Spezialdiät umzusetzen. Ein Agitationsspektakel im Sinne von Michael Moore, den er als seinen Lehrer betrachtet, eine ätzende Kritik an der Fast-food-Industrie, die mit einem millionenschweren Budget Kinder und Jugendliche zur Fehlernährung verführt. Beeindruckend ist sein Leidensweg, der die Feuerbachsche These, wonach der «Mensch ist, was er isst», minutiös belegt. Er nimmt um $12 \mathrm{~kg} \mathrm{zu}$, Blutdruck, Cholesterinwerte und Lebertransaminasen machen ihn zum Selbstmordkandidaten. Er wird reizbar, depressiv, impotent und lethargisch, leidet an Kopfschmerzen und Atemnot, wird süchtig nach seiner täglichen Kohlenhydrat-Fett-Mast. Die landesweite Fettepidemie wird mit Zahlen und Fakten belegt. Spurlock untermauert seine Völlerei mit Interviews vor laufender Kamera. Konsumenten, Schüler und Eltern, Patienten, Verkäufer, Schulköche, Lehrer und Pressesprecher der Industrie sind Statisten seines Kreuzzuges. Er recherchiert die Werbemethoden, die Produkte, den Umsatz und die Folgen übermässigen Konsums bis hin zur Gastric-banding-Operation. Schrill, schräg und genau, polemisch, karikierend, vereinfachend und konsequent.

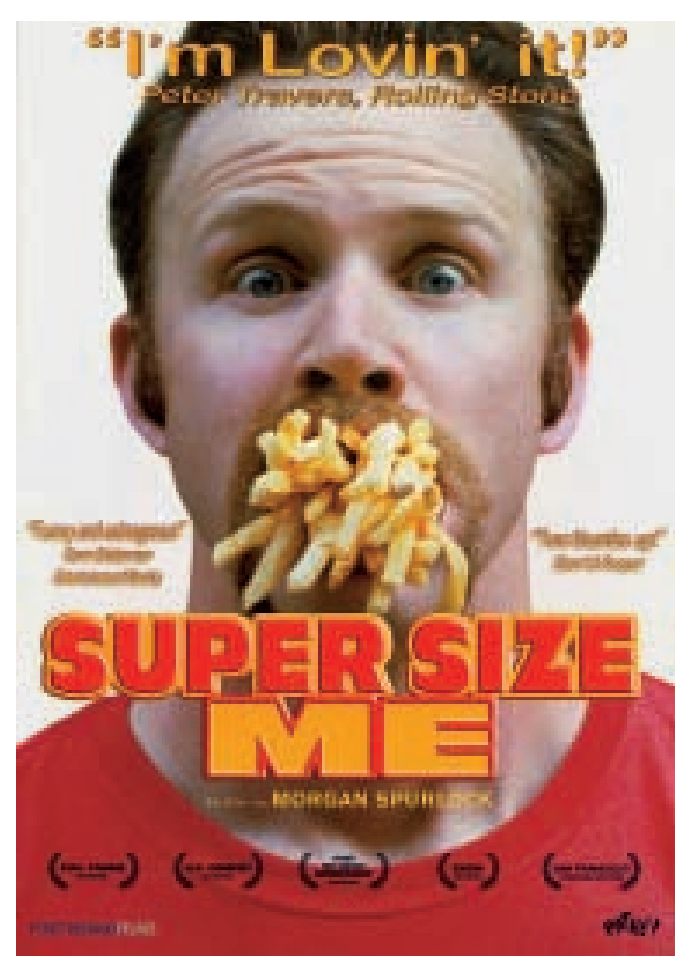

Sein Spektakel forscht nicht in die Tiefe, es simplifiziert nach der Schwarzweissmethode die bekannte Botschaft von: zu fett - zu süss - zu viel. Spurlock hat sich nach vollbrachter Tat durch seine veganische Freundin entgiften lassen. Rund ein Jahr dauerte das Abspecken bis zum Ausgangsgewicht. Das Abenteuer hat nicht nur ihm, sondern auch McDonald's einige Kopfschmerzen verursacht.

\section{Die Reaktionen}

Schon fünf Wochen nach dem Kinostart hatte das Filmtagebuch das Hundertfache der Herstellungskosten eingespielt. Vor allem Teenager, das Zielpublikum der Fast-food-Giganten, reagieren zustimmend. Australische Kinobesitzer beklagen sich über mehr Reinigungsarbeiten, weil die Zuschauer ihr Popcorn und Cola liegenlassen. Mehrere Schulen haben ihren Ernährungsplan umgestellt. Umsatzeinbussen waren für McDonald's die schmerzliche Folge. Ein gegenüber dem Regisseur allzu freimütiger Lobbyist wurde entlassen. Obwohl die Auswirkungen des Films heruntergespielt werden, hat 
sich das Angebot an Gemüse und Früchten in den USA plötzlich verbessert. Der Hersteller sponsert Sportveranstaltungen und verteilt Kalorientabellen. Pech nur, dass der Marketingleiter der Gegenoffensive, kurz nach Beginn an einem Herzinfarkt verstarb, was fatal an die Glacehersteller im Film erinnert, die ihren Verkaufserfolg mit Diabetes und verstopften Kranzarterien bezahlten. Mehrere «Übergrössen» wurden aus dem Sortiment entfernt, sie ersetzt ein «Go Active Happy Meal», inklusive Schrittzähler und Gymnastikprogramm. «Who has the gold, has the rule», wer Geld hat, hat auch Experten. Der renommierte «American Council on Science and Health» organisierte Ernährungsberater, die den Film in TV-Spots abqualifizierten, den sie als irreführend, gefährlich und ekelhaft einstuften.
Nachdem die Presse Burger King, McDonald's, Coca Cola, ExxonMobil und General Motors als Finanzquellen dieses Councils offenlegte, hat sich der Ton der Gegenpropaganda deutlich gemässigt.

Jeder schweizerische Ernährungsbericht bestätigt den Fachleuten die Zunahme der Fettepidemie. Ausserhalb dieser Kreise bewegen die Zahlen aber wenig. Das BAG versucht, mit «Suisse Balance» eine grössere Breitenwirkung zu erreichen. Gegen Überkonsum, Fehlernährung und Bewegungsmangel soll die Adresse www. suissebalance.ch weiterhelfen. Vom Aufwind durch einen Kassenhit wie «Super size me» können Konsumenten und Gesundheitsstrategen hierzulande nur träumen.

\section{Geschichten, die kein Code erfasst}

\section{B. Gurtner}

\section{ICD 10 - I21.0}

Der 42jährige Aussendienstvertreter einer grossen Lebensversicherungsgesellschaft wurde direkt aus dem Seminarraum eines Hotels eingeliefert, in dem die Quartalskonferenz der regionalen Agenturleiter im Gange war. An diesen Meetings war es üblich, die Leistungszahlen der einzelnen Mitarbeiter von transparenten Folien grell an die Wand zu projizieren. Die Guten erhielten Lob und einen Bonus, die Versager hämischen Tadel und eine letzte Chance, es besser zu machen. Der beruflich wenig erfolgreiche Patient hatte wegen Erwartungsangst in den Nächten zuvor kaum geschlafen und innert zweier Tage 60 Zigaretten geraucht. Vor all seinen Kollegen blamiert, brach er zusammen. Die Direktion der lebensversichernden Gesellschaft sah sich nach diesem Infarkt zu keiner Kursänderung veranlasst.

\section{ICD 10 - I21.4}

Der 28jährige Lastwagenfahrer aus Polen verbrachte die kalte Winternacht auf einem Rastplatz in seiner Führerkabine, um Hotelspesen zu sparen. Er rauchte viel und liess die Fahrzeugheizung eingeschaltet, nicht aber die Ventilation. Akute pektanginöse Beschwerden und
Lufthunger gegen 5 Uhr. Glück im Unglück durch eine gleichzeitige Kontrolle der Autobahnpolizei. Kohlenmonoxydvergiftung mit anoxämisch bedingtem Herzinfarkt.

\section{ICD 10 - 121.1}

1. August. Heisser Tag. Internes Klubturnier auf dem Tennisplatz. Der 55jährige ehrgeizige Bankprokurist will es den Jungen nochmals zeigen. Spielt bis zur Erschöpfung, kollabiert. Der Herzinfarkt beendet seine sportliche und berufliche Karriere.

\section{ICD $10-121.3$}

Der 49jährige sehr kräftige Metzger wird von einer Wespe mit einem Stich in den Nacken gefällt. Schwerer allergischer Schock. Sekundärer Herzinfarkt.

\section{ICD $10-121.2$}

Der 44jährige übergewichtige Büroangestellte war Vorstandsmitglied in vielen Klubs und Vereinen. Am Grümpelturnier blieb er nach einem ruppigen Zweikampf mit einem Knöchelbruch 
liegen. Im Spital schweres Alkoholentzugsdelirium, in dessen Verlauf letaler Herzinfarkt. Die Trauerfeier in der überfüllten Dorfkirche glich einem Staatsbegräbnis.

\section{ICD 10 - 121.9}

Turnusgemäss wäre es Aufgabe des Nachbarn gewesen, den Schnee von der Zufahrt zur Tiefgarage wegzuräumen. Einmal mehr drückte er sich, weshalb der pensionierte Lehrer frühmorgens in beissender Kälte zur Schaufel griff, um noch vor dem Frühstück die Rampe freizulegen. Der frischgefallene nasse Schnee war schwer, der Ärger gross, der Blutdruck stieg und der Nüchternglukosespiegel sank. Typischer Schneeschauflerinfarkt.

\section{ICD 10 - 122.9}

72jähriger Witwer, nach einem ersten Infarkt unter Aspirin und wegen VH-Flimmerns dauerantikoaguliert. Bactrimstoss gegen Harnwegsinfekt, dadurch Potenzierung des Marcoumars. Quickabfall auf $12 \%$. Reinfarkt wegen schwerer Anämie. Massive Melaina erst zwei Tage später infolge eines zuvor symptomlosen präpylorischen Magenulkus.

\section{ICD $10-121.2$}

(fiktiv) Der 57jährige Allgemeinpraktiker ist um 23.15 Uhr in seiner Praxis nach einem Wutanfall wegen unlösbarer Probleme mit der Codiersoftware unsanft dahingegangen. An der Herbstversammlung der regionalen Ärztegesellschaft wird man das einst verdienstvolle Mitglied während einer Schweigeminute von 18 Sekunden Dauer gebührend ehren.

$\mathrm{Zu}$ gedenken ist auch der für Notfalldienste verlorengegangenen Kolleginnen und Kollegen, die sich in das patientenferne Grenzgebiet zwischen Medizin und Ökonomie begeben haben und dort als spezialisierte Codierer das lebendige Spitalgeschehen in tote Zahlen pressen müssen. Ihre wohl etwas frustrierende Arbeit entlastet die Kliniker von einer ungeliebten Büropflicht und schenkt diesen wertvolle Zeit für mehr Patientenkontakte. Hoffentlich schaffen es die dem eigentlichen Arztberuf entsagenden Klassifizierkünstler, ohne ICD 10 F33.9 über die Runden zu kommen. Wenn sie in den geschichteten Krankenakten oder in den Austrittsberichten mit Datasklerose doch noch einige Anamnesen finden, fehlt es ihnen ja nicht an spannender Lektüre. 